ARTIGOS

\title{
COMO APRENDEM OS ESTUDANTES E PROFESSORES DE UMA INSTITUIÇÃO DE ENSINO SUPERIOR: APLICAÇÃO DO INVENTÁRIO DE ESTILOS DE APRENDIZAGEM DE KOLB (1984)
}

\author{
HOW STUDENTS AND TEACHERS FROM A \\ HIGHER EDUCATION INSTITUTION LEARN: \\ APPLICATION OF KOLB'S LEARNING STYLES \\ INVENTORY (1984)
}

\begin{abstract}
José Adailton de Abreu dailtonabr@gmail.com Bacharelando em Administração na Universidade de Pernambuco (UPE) em Salgueiro. Salgueiro $P E-B R$.
\end{abstract}

\author{
Josiete da Silva Mendes \\ josiete.mendes@upe.br \\ Mestra em Administração \\ (UFPB). Professora Assistente \\ da Universidade de Pernambuco \\ (UPE) - campus Salgueiro. \\ Salgueiro - PE - BR.
}

\begin{abstract}
Maria Edilene de Oliveira edileneoliveira9816@gmail.com Bacharelanda em Administração na Universidade de Pernambuco (UPE) em Salgueiro. Salgueiro $P E-B R$.
\end{abstract}

Tatyane Veras de Queiroz Ferreira da Cruz tatyane.cruz@upe.br Doutoranda em Psicologia Cognitiva (UFPE). Professora Assistente da Universidade de Pernambuco (UPE) - campus Salgueiro. Salgueiro - PE - BR.

Wanderberg Alves Brandão wanderberg.brandao@upe.br Mestre em Administração (UFPB). Professor Assistente da Universidade de Pernambuco (UPE) - campus Salgueiro. Salgueiro - PE - BR.

\section{RESUMO}

Aprender e ensinar faz parte da vida do ser humano, constituindo essas ações como um complexo sistema de interação entre professor e estudante dentro do processo de ensino e aprendizagem. Com base nisso, este trabalho analisou como aprendem os membros de uma comunidade acadêmica baseado na teoria de Kolb por meio de uma pesquisa quantitativa descritiva transversal, processando os dados obtidos com o uso de técnicas estatísticas descritivas. Os dados apontaram que os discentes e docentes possuem, como estilo de aprendizagem predominante, o assimilador, estando mais envolvidos com modelos teóricos do que com a vivência prática. Acredita-se que a compreensão dos estilos de aprendizagem enriquece a prática acadêmica, viabilizando o aprendizado, de forma mais significativa, de acordo com o perfil dos estudantes. Ademais, considera-se que a sintonia entre os estilos de aprendizagem dos discentes e docentes parecem contribuir para o bom desempenho desses estudantes.

Palavras-chave: Estilos de Aprendizagem. Inventário de Kolb. Educação Gerencial.

\begin{abstract}
Learning and teaching are part of human life, constituting these actions as a complex system of interaction between teacher and student within the teaching-learning process. Based on this, this work analyzed how members of an academic community learn based on Kolb's theory through a cross-sectional quantitative research, processing the data obtained using descriptive statistical techniques. The data showed that students and teachers have the assimilator as the predominant learning style, being more involved
\end{abstract}


with theoretical models than with practical experience. It is believed that the understanding of learning styles enriches academic practice, enabling learning possible in a more meaningful way, according to the profile of students. Furthermore, it is considered that the harmony between the learning styles of students and teachers seems to contribute to the good performance of these students.

Keywords: Learning Styles. Kolb Inventory. Management Education.

\section{INTRODUÇÃO}

Constantemente, os discentes indagam sobre a falta de prática que proporcione uma aprendizagem experimental, enquanto os professores argumentam que os alunos não conseguem compreender os conceitos e os fundamentos das disciplinas (VALENTE; ABIB; KUSNIK, 2007). Compreender os estilos de aprendizagem, que são as maneiras como os indivíduos processam a informação, os sentimentos e os comportamentos, em situações de aprendizagem, torna-se necessário para potencializar o processo de ensino.

Não verificar o estilo de aprendizagem do aluno e ensinar utilizando sempre as mesmas estratégias, exercícios e procedimentos, sem considerar os interesses dos estudantes, pode provocar o desinteresse, a desmotivação e não contempla as possibilidades de aprendizagem (DIAS; SAUAIA; YOSHIZAKI, 2013). Por isso, é fundamental analisar o processo de ensinar e aprender.

Assim, esse interesse em avaliar os membros de uma comunidade acadêmica emergiu por considerar relevante a identificação dos estilos de aprendizagem de ambos, visto que os docentes tendem a ensinar da maneira como aprendem, e que tais estilos repercutem no fazer pedagógico. Reconhecer o estilo de aprendizagem do docente possibilita a ele ampliar seu olhar para outras estratégias e métodos de ensino, que não sejam apenas aquelas relacionadas com sua forma de aprender, mas aquelas que atendam tam- bém às necessidades dos estudantes. Acredita-se que, dessa maneira, os resultados desta pesquisa darão um novo sentido ao processo de ensino e aprendizagem, nesta instituição.

Diante desse cenário, buscou-se, por meio da aplicação do Inventário de Estilo de Aprendizagem (KOLB, 1984), entender como estudantes e professores aprendem no âmbito da Universidade de Pernambuco (UPE) - Campus Salgueiro. O Inventário de Estilo de Aprendizagem (Learning Style Inventory - LSI) de Kolb foi criado em 1984 e fundamenta-se no ciclo de aprendizagem, que acontece a partir da experiência, e oferece quatro estilos de aprendizagem, dando suporte para analisar os diferentes perfis que distinguem as pessoas nesse processo de ensino e aprendizagem.

Afora o modelo proposto por Kolb (1984), foram criados outras teorias e métodos que avaliam conjuntos de dimensões distintos, mas que podem ter nomenclaturas diferentes para perspectivas similares. Citam-se modelos como VARK (FLEMING; MILLS, 1992), Felder e Silverman (1988) e Dunn e Dunn (1978). O Modelo do Estilo de Aprendizagem VARK, desenvolvida por Fleming e Mills (1992), propõe cinco características de destaque nos indivíduos: visual; auditivo; leitor/escrito; cinestésico; e multimodal. Já Felder e Silverman (1988) considera que, no processo de aprendizagem, existem duas etapas: recepção e processamento da informação, e pontuou cinco dimensões: (1) Processamento: ativo ou reflexivo; (2) Percepção: sensorial ou intuitiva; (3) Entrada/Retenção: visual ou verbal; (4) Compreensão: sequencial ou global; e (5) Organização: indutiva ou dedutiva. Anos depois, Felder e Soloman (1991) desenvolveram, a partir desse modelo, um instrumento chamado ILS (Index of Learning Styles), para identificar os estilos de aprendizagem dos alunos dentro dessas dimensões. Por último, o modelo proposto por Dunn e Dunn (1978) define o estilo de aprendizagem como a forma em que os indivíduos se concentram para internalizar e reter informações novas. Sendo assim, pondera as dimensões que podem interferir no processo de 
aprendizagem: estímulos ambientais, emocionais, sociais, físicos e psicológicos.

O LSI, de Kolb (1984), é o inventário mais utilizado em pesquisas nacionais e internacionais com universitários e possui maior aplicação e divulgação. Ademais, tem sua confiabilidade e validade testada e confirmada (SCHMITT; DOMINGUES, 2016). Por isso, esse trabalho fundamenta-se a partir do LSI, de Kolb (1984), o qual aponta para quatro estilos de aprendizagem: (1) Acomodador; (2) Divergente; (3) Convergente; e (4) Assimilador. Esses estilos individuais de aprendizagem de Kolb (1984) foram criados por meio da combinação (dois a dois) dos quatro estágios de aprendizagem: (1) experiência concreta (EC); (2) observação reflexiva (OR); (3) conceituação abstrata (CA); e (4) experimentação ativa (EA) (SILVA et al., 2012) resultando, assim, em "Divergente $=\mathrm{EC}+\mathrm{OR}$; Assimilador $=\mathrm{CA}$ + OR; Convergente $=\mathrm{CA}+\mathrm{EA}$; e Acomodador $=\mathrm{EA}+\mathrm{EC}$ " (SILVA et al., 2012, p. 26).

Entende-se que, por meio da descoberta dos estilos de aprendizagem dos discentes e docentes, podem-se subsidiar atividades que atendam às necessidades de aprendizagem dos alunos, bem como, por meio das diferenças entre o modo de ensinar dos docentes, conduzir atividades de ensino institucionalmente apropriadas.

Assim, como relevância prática deste artigo, acredita-se que, por meio dos subsídios teóricos e empíricos apresentados, os gestores das instituições de ensino podem refletir e agir em relação às práticas de ensino adotadas pelos docentes e redirecionar esforços em programas de capacitação e treinamento que consigam abranger uma amplitude de estilos de aprendizagem dos discentes.

No que se refere à relevância teórica do estudo, somam-se aos demais realizados na área (VALENTE; ABIB; KUSNIK, 2007; MOREIRA; MUNCK, 2010; TREVELIN, 2011; REIS; PATON; NOGUEIRA, 2012; SILVA et al., 2012; OLIVEIRA et al., 2013; BRANDÃO, 2014; PENA; CAVALCANTE; MIONI, 2015; OLIVEIRA; BOUZADA, 2018) no sentido de contribuir para o entendimento dos diferentes estilos de aprendizagem dos discentes e docentes das instituições de ensino superior do Brasil.

Desse modo, questiona-se nesta pesquisa: qual o estilo de aprendizagem predominante entre os discentes e docentes da Universidade de Pernambuco (UPE) - Campus Salgueiro? Existe compatibilidade entre os estilos dos discentes e docentes? Para tanto, buscou-se, a partir da aplicação do inventário de Kolb, identificar o estilo de aprendizagem de discentes e docentes do Campus da UPE em Salgueiro. Salienta-se que este estudo se desenvolve em uma das unidades da Universidade, que conta ainda com outros 10 (dez) campi em todas as regiões do território pernambucano.

$\mathrm{Na}$ sequência, apresenta-se a fundamentação teórica com o embasamento deste estudo descrevendo, de forma detalhada, o modelo de estilo de aprendizagem escolhido para esta pesquisa; a seguir, mostram-se os procedimentos metodológicos e a análise dos dados. E, na última seção, as considerações finais desta pesquisa.

\section{FUNDAMENTAÇÃO TEÓRICA}

As pessoas são diferentes, e a forma como enxergam o mundo influencia diretamente seus processos de aprendizagem. Assim, é importante questionar se, diante dessa diversidade, pode-se estabelecer um único formato de ensino. Pontua-se que diversos padrões de aprendizagem precisam ser considerados, pois cada pessoa apresenta sua forma de processar informação, conhecimento e perceber as situações no contexto de aprendizagem. Desse modo, acredita-se que não é possível ensinar de uma única forma, pois os indivíduos são diferentes em suas particularidades.

O modelo dos estilos de aprendizagem considera essa diversidade em todo processo, na forma que age e interpreta a realidade para alcançar o conhecimento. Para Valente, Abib e Kusnik (2007, p. 55) "a evolução dos estudos sobre como as pessoas aprendem passa por identificar os estilos de aprendizagem com que cada um nasce e/ou desenvolve durante toda a sua vida". Ainda de acordo com os autores su- 
pracitados, "a maior parte dos indivíduos possui entre 6 (seis) e 14 (quatorze) preferências que constituem seu estilo de aprendizagem. Quanto mais forte for determinada preferência, mais importante será atendê-la." (VALENTE; ABIB; KUSNIK, 2007, p. 56).

Diante desse cenário, Kolb (1984) criou um modelo teórico (Inventário do Estilo de Aprendizagem - Learning Style Inventory - LSI) para identificar os estilos de aprendizagem, baseando-se no ciclo de aprendizagem composto por quatro estágios e permeado pela experiência: (1) Acomodador; (2) Divergente; (3) Convergente; e (4) Assimilador. Na sequência, as variáveis/estágios do modelo são explicadas.

\subsection{O INVENTÁRIO DOS ESTILOS DE APRENDIZAGEM DE KOLB}

O indivíduo que possui o estilo de aprendizagem "Divergente" tem como característica ser muito observador, sendo uma pessoa que gosta de ouvir e, posteriormente, compartilhar suas ideias. Para Pena, Cavalcante e Mioni (2015, p. 70), os divergentes "tendem a afastar-se das soluções convencionais, e optar por possibilidades alternativas, preferindo discussões, produção de ideias e trabalhos em grupo." Pode-se citar como perguntas preferidas das pessoas do estilo divergente: (a) Por que as pessoas que trabalhavam na indústria no periodo da Revolução Industrial sob os ensinamentos de Taylor se sentiam desmotivadas ou deprimidas? (b) Por que a questão salarial dos funcionários não é o único fator decisivo para a motivação funcional nas empresas?

Aqueles que possuem o estilo de aprendizagem "Assimilador" são dedutivos e apropriam-se de modelos teóricos para chegar a resultados práticos. Sendo a teoria o mais importante no seu modo de aprender (PENA; CAVALCANTE; MIONI, 2015). Para as pessoas assimiladoras, pode-se citar como exemplos de perguntas preferidas: (a) O que ocorre para que os carros sejam produzidos mais rápidos e a um custo muito menor nas indústrias Ford? (b) O que se pode fazer para reduzir os custos de produção de carros elétricos?

De acordo com Pena, Cavalcante e Mioni (2015, p. 70), o estilo de aprendizagem "Acomodador" "possui duas preferências de aprendizagem baseadas na experimentação ativa e na experiência concreta, ou seja, tendem a priorizar seus sentimentos em suas tomadas de decisão". As pessoas que possuem esse estilo, "seguem mais seus instintos do que teorias e estudos lógicos, mas confiam nos outros para realizar análises mais complexas de relatórios." (OLIVEIRA; BOUZADA, 2018, p. 5).

Já para as pessoas do estilo Acomodador, tem-se como perguntas favoritas: (a) $E$ se, nas Indústrias Ford, no período da Revolução Industrial, fossem inseridos programas de incentivo salarial e séries de lazer, os sintomas de desmotivação e da depressão seriam reduzidos? (b) E se o Brasil reduzisse os impostos da folha de pagamento funcional, ocorreria redução do desemprego?

Os resultados do estudo de Valente, Abib e Kusnik (2007) apontaram que, entre os discentes, o estilo predominante é o Acomodador (58\%), já os docentes apresentam maior incidência do estilo Convergente (45\%).

Nas pessoas com o estilo de aprendizagem "Convergente", é possível verificar que existe uma preferência por modelos teóricos; porém, diferente dos assimiladores, opta-se por conceitos que possam ser utilizados na prática. São indivíduos que "normalmente usam o raciocínio dedutivo para aplicar suas ideias e costumam ser bastante produtivos na definição de problemas." (OLIVEIRA; BOUZADA, 2018, p. 5). Entre as perguntas favoritas das pessoas convergentes, pode-se citar: (a) Como a introdução da linha de produção por Henri Ford foi capaz de reduzir os custos de produção dos carros? (b) Como a alta do dólar pode ser benéfica para alguns setores da economia brasileira?

$\mathrm{Na}$ pesquisa realizada por Pena, Cavalcante e Mioni (2015) com estudantes do curso de Administração do Centro Universitário FECAP, chegou-se à conclusão de que o estilo convergente é predominante entre os discentes, com $41 \%$ de respostas apontadas nesse sentido. 
O Quadro 1 sintetiza como se norteiam os estilos de aprendizagem.

Quadro 1 - Características dos estudantes por meio do Inventário dos Estilos de Aprendizagem de Kolb

\section{Estudante Divergente}

\section{Questão favorita: Por quê?}

Integra experiência com seus próprios valores e sentimentos

Prefere ouvir e partilhar ideias, aprendendo pela experiência concreta e a observação reflexiva

Criativo e inovador, tem facilidade para propor alternativas, reconhecer problemas e compreender as pessoas

Gosta de saber o valor do que irá aprender

\section{Estudante Assimilador}

\section{Questão favorita: O quê?}

Integra experiência com conhecimentos já existentes

Conceitualizador, utiliza dedução para resolver problemas

Trabalha bem com muitos detalhes e dados, dando-lhes uma organização lógica

Procura assimilar novas ideias e pensamentos

Interessados mais pela lógica de uma ideia do que pelo seu valor prático

\section{Estudante Convergente}

\section{Questão favorita: Como?}

Integra teoria e prática

Utiliza tanto a abstração como o senso comum na aplicação prática das ideais e teorias

Gosta de resolver problemas práticos e tem bom desempenho nos testes convencionais

Procura sempre as soluções ótimas para os problemas práticos

Combina a dedução e a indução na solução de problemas

\section{Estudante Acomodador}

\section{Questão favorita: E se?}

Integra experiência com aplicação e faz imediata aplicação da nova experiência

Utiliza a indução na resolução de problemas

Aprende por ensaio e erro e, frequentemente, descobre o novo conhecimento sem a ajuda do professor

Altamente ativo e criativo, adapta-se facilmente a novas situações

Independente, líder natural

Fonte: adaptado de Valente, Abib e Kusnik (2007).

Outras pesquisas de natureza qualitativa (TREVELIN, 2011; OLIVEIRA; BOUZADA, 2018), apesar de não seguirem à risca o modelo teórico de Kolb (1984), apontaram os seguintes resultados: Trevelin (2011) pesquisou a relação de discentes da Faculdade de Tecnologia de Taquaritinga com um determinado professor, chegando à conclusão de que a diferença entre os estilos de aprendizagem do docente e dos discentes influencia sua relação com a turma. Já Oliveira e Bouzada (2018) buscaram entender se, em trabalhos de equipes, a diferença entre estilos de aprendizagem sugere interferências no processo de ensino e aprendizagem, chegando a conclusões negativas para essas suposições.

Pode-se notar que, na literatura, especializada não há um consenso acerca do estilo de aprendizagem predominante entre os docentes ou discentes, variando, principalmente, entre os cursos.

\section{PROCEDIMENTOS ME- TODOLÓGICOS}

A presente pesquisa enquadra-se como 
de abordagem quantitativa, sendo sua natureza descritiva com corte transversal, na medida em que a amostra é consultada somente uma vez. Como forma de levantamento dos dados, foi aplicado o Inventário de Estilo de Aprendizagem idealizado por Kolb (1984) em uma amostra de 110 (cento e dez) discentes da Universidade de Pernambuco (UPE) - Campus Salgueiro, que representa $73,3 \%$ de uma população de cerca de 150 (cento e cinquenta) estudantes distribuídos no Curso Superior de Bacharelado em Administração e Superior de Tecnologia em Logística. A coleta de dados aconteceu nos meses de junho e julho de 2019.

Com relação ao grupo de estudantes que responderam à pesquisa, 58,2\% eram do sexo feminino, enquanto $41,8 \%$ eram do sexo masculino. A categorização dos estudantes por turma/curso está descrita na tabela 1 , a seguir.

Tabela 1 - Respondentes discentes por turma

\begin{tabular}{ccc}
\hline Turma & Número de discentes & Porcentagem \% \\
\hline $2^{\text {o }}$ Período - Administração & 38 & 34,55 \\
\hline $4^{\circ}$ Período - Administração & 21 & 19,09 \\
\hline $6^{\circ}$ Período - Administração & 22 & 20,00 \\
\hline $8^{\circ}$ Período - Administração & 21 & 19,09 \\
\hline Logística & 8 & 7,27 \\
\hline Total & 110 & $100 \%$ \\
\hline
\end{tabular}

Fonte: elaborado pelos autores (2019).

Visando comparar os estilos de aprendizagem de discentes e docentes, aplicou-se, também, o mesmo instrumento entre os docentes da instituição, que contava, no período da pesquisa, com nove professores no quadro efetivo; entretanto, dessa população, sete responderam, o que representa uma amostra de 77,8\%. Dos docentes respondentes, $42,9 \%$ eram do sexo feminino, enquanto $57,1 \%$ eram do sexo masculino.

$\mathrm{O}$ processo de pesquisa foi realizado da seguinte forma: (1) inicialmente, enviou-se para os discentes e docentes via WhatsApp $\mathbb{R} \mathrm{e}$ e-mail o link do site com o instrumento (CCMD -UFPB, 2019); (2) na sequência, os participantes responderam com o print ou foto da tela que constava o resultado; (3) por fim, os resultados foram tabulados e analisados. Para análise dos dados, optou-se pelo uso de medidas descritivas que foram tabuladas e analisadas com o uso do Microsoft Office Excel ${ }^{\circledR}$.

\section{ANÁLISE DOS DADOS}

\subsection{COMO OS DISCENTES APRENDEM}

Conforme se apresenta na tabela 2, a maioria dos discentes da UPE - Campus Salgueiro, 40\% dessa amostra, possui o estilo de aprendizagem “Assimilador". De acordo com Kolb (1984), os indivíduos com essa característica preferem aprender por meio de modelos teóricos, sem relacionamento direto com a experiência prática. O presente resultado é semelhante ao de Souza et al. (2013) que apresentou $46 \%$ dos estudantes da amostra de um curso de Administração como assimiladores, e o de Cordeiro e Silva (2012), que também identificaram o estilo de aprendizagem assimilador como predominante entre estudantes do curso de administração, respondendo por $38,7 \%$ da amostra. Cerqueira (2000), que analisou o perfil de estudantes de várias áreas de conhecimento, igualmente identificou que o perfil assimilador era o de maior predominância, o que indica que essa pode ser uma tendência de áreas diversas entre os estudantes brasileiros.

Para os estudantes com o estilo de aprendizagem Assimilador, "o professor deve exercer o papel de um especialista, auxiliando-o na compreensão das informações e na construção lógica do problema" (OLIVEIRA; BOUZADA, 2018, p. 5). Oliveira e Bouzada (2018) 
afirmam que o perfil assimilador pode ter dificuldades de se relacionar com os outros estilos, devido à falta da experimentação ativa, por preferir criar modelos abstratos e teóricos para solucionar problemas. Os autores supracitados também alertam que os pertencentes a esse estilo de aprendizagem podem ser incapazes de aplicar seus conhecimentos a situações práticas, o que é negativo no processo de ensino e aprendizagem.

Tabela 2 - Distribuição dos estilos de aprendizagem por turma

\begin{tabular}{cccccc}
\hline Turma & Divergente & Assimilador & Convergente & Acomodador & Porcentagem \\
\hline $2^{\text {o }}$ Período - & $\mathbf{2}$ & $\mathbf{1 3}$ & $\mathbf{1 3}$ & $\mathbf{1 0}$ & $\mathbf{3 8}$ \\
Administração & $1,82 \%$ & $11,82 \%$ & $11,82 \%$ & $9,09 \%$ & $34,55 \%$ \\
\hline $4^{\mathbf{o}}$ Período - & $\mathbf{1}$ & $\mathbf{1 0}$ & $\mathbf{5}$ & $\mathbf{5}$ & $\mathbf{2 1}$ \\
Administração & $0,91 \%$ & $9,09 \%$ & $4,55 \%$ & $4,55 \%$ & $19,09 \%$ \\
\hline $6^{\mathbf{0}}$ Período - & $\mathbf{1}$ & $\mathbf{1 0}$ & $\mathbf{9}$ & $\mathbf{2}$ & $\mathbf{2 2}$ \\
Administração & $0,91 \%$ & $9,09 \%$ & $8,18 \%$ & $1,82 \%$ & $20,00 \%$ \\
\hline $8^{\text {o }}$ Período - & $\mathbf{2}$ & $\mathbf{8}$ & $\mathbf{7}$ & $\mathbf{4}$ & $\mathbf{2 1}$ \\
\cline { 2 - 5 } Administração & $1,82 \%$ & $7,27 \%$ & $6,36 \%$ & $3,64 \%$ & $19,09 \%$ \\
\hline Logística & $\mathbf{0}$ & $\mathbf{3}$ & $\mathbf{5}$ & $\mathbf{0}$ & $\mathbf{8}$ \\
\hline Total & $0,00 \%$ & $2,73 \%$ & $4,55 \%$ & $0,00 \%$ & $7,27 \%$ \\
\hline
\end{tabular}

Fonte: elaborado pelos autores (2019).

O segundo estilo com maior predominância foi o "convergente", que retrata características do perfil do administrador (PENA; CAVALCANTE; MIONI, 2015). Os indivíduos pertencentes a essa categoria buscam aplicar a teoria à prática, mas tendo como prioridade os aspectos teóricos. Nesta amostra, 35,45\% demonstram ter também esse estilo. Esse dado torna-se relevante porque condiz com o perfil da formação superior desses estudantes. Ressalta-se que, segundo Kolb (1984), todos os indivíduos possuem os quatro estilos de aprendizagem, sendo o teste uma perspectiva da predominância e não de sua totalidade.

O terceiro estilo mais frequente da amostra pesquisada é o acomodador, o qual busca o aprendizado por meio da prática (KOLB, 1984), corroborando a pesquisa de Moreira e Munck (2010), que, embora em contexto diferente (análise da satisfação entre os estilos individuais de aprendizagem e o treinamento vivencial ao ar livre), apontou que o estilo acomodador é o que apresenta a maior satisfação ao final das atividades práticas.
Por fim, aproximadamente $6 \%$ da amostra pesquisada identificaram-se como divergente, destacando que, no Curso de Tecnologia em Logística, este estilo não foi observado em nem um dos participantes. O estudo de Reis, Paton e Nogueira (2012) também identificou esse estilo como o de menor predominância no curso de Ciências Contábeis entre instituições públicas e privadas.

Segundo Oliveira e Bouzada (2018, p. 4), os divergentes "são pessoas que preferem ver situações concretas de diferentes pontos de vista, e, normalmente, dão-se melhor com atividades mais criativas porque buscam pensar fora da caixa.". Dessa forma, parece que esse estilo não está presente nesses estudantes de forma ativa.

Acerca da distribuição dos estilos de aprendizagem com relação à variável "sexo" dos estudantes respondentes, percebe-se, por meio do gráfico 1, uma similaridade na forma como estão distribuídos os estilos de aprendizagem, tanto no sexo feminino, quanto no masculino. 
Gráfico 1 - Comparação entre Sexo x Estilo de aprendizagem

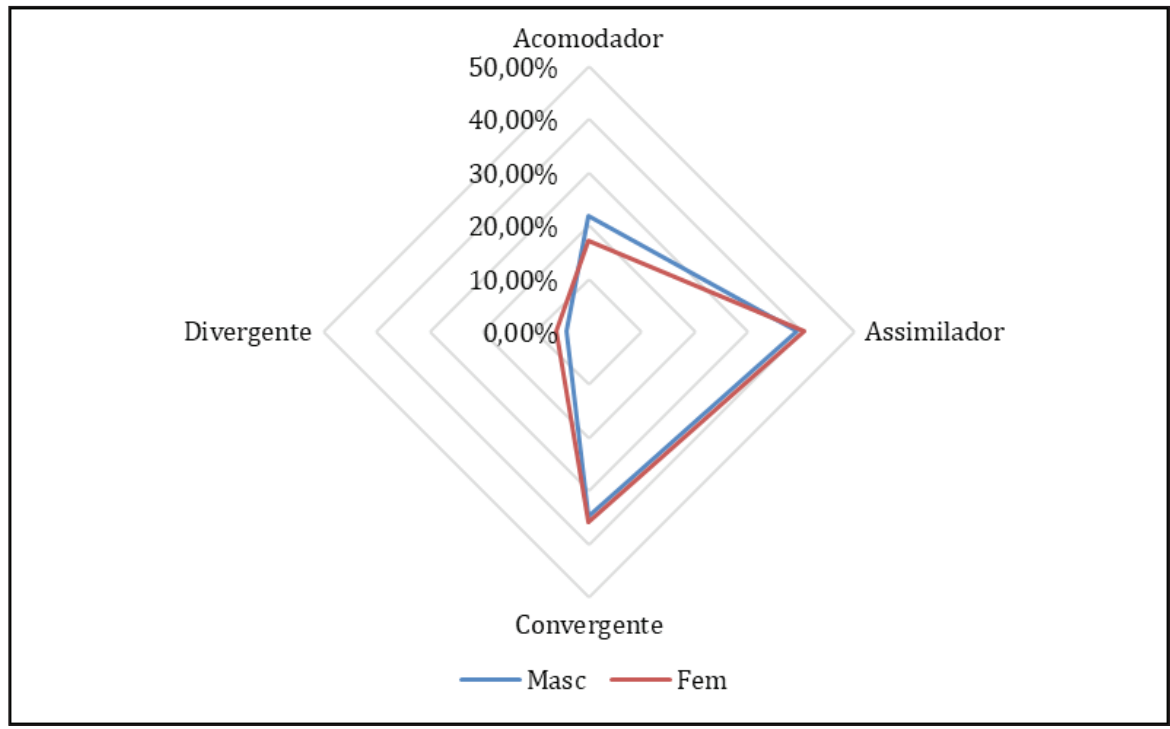

Fonte: Elaborado pelos autores (2019).

Em outras palavras, percebe-se que há ligeira diferença entre a distribuição dos estilos de aprendizagem quando se isola a variável sexo, sendo, proporcionalmente, a distribuição do perfil de aprendizagem de ambos os sexos parecida. Esse resultado está de acordo com os achados na pesquisa de Oliveira et al. (2013), uma vez que a variável sexo não foi determinante para a definição do estilo de aprendizagem predominante.

\subsection{COMO OS DOCENTES APRENDEM}

$\mathrm{Na}$ amostra desta pesquisa, a maioria $(42,8 \%)$ dos docentes apresentaram o estilo de aprendizagem assimilador, seguido dos estilos Acomodador e Convergente, ambos com 28,5\% cada. Para Pena, Cavalcante e Mioni (2015), os indivíduos da área de humanas caracterizam-se como assimiladores ( $36 \%$ da amostra). Como o campo da Administração está incluído nas ciências sociais aplicadas, os dados apontam para características semelhantes.

Tabela 3 - Distribuição dos estilos de aprendizagem por docente

\begin{tabular}{ccc}
\hline Estilo de aprendizagem predominante & Quantidade & Porcentagem \\
\hline Assimilador & 3 & $42,86 \%$ \\
\hline Acomodador & 2 & $28,57 \%$ \\
\hline Convergente & 2 & $28,57 \%$ \\
\hline Divergente & 0 & $0 \%$ \\
\hline Total & 7 & $100 \%$ \\
\hline
\end{tabular}

Fonte: elaborado pelos autores (2019).

Esses dados identificam que os estilos de aprendizagem dos docentes e discentes seguem o mesmo perfil, estando a maioria com predominância do estilo assimilador. Embora esse trabalho discorra sobre a incidência de um estilo de aprendizagem dos docentes, torna-se relevante pontuar que, de acordo com Trevelin (2011), o ideal é que os professores trabalhem o processo de ensino e aprendizagem passando por todos os quatro quadrantes do ciclo de aprendizagem, para atingir a todos os discentes. Espera-se do docente que seja capaz de expli- 
car um problema de diversas maneiras, favorecendo a aprendizagem dos estudantes, nos mais variados estilos de aprendizagem.

Assim, a maneira de apresentar um conteúdo aos estudantes por parte do professor deve percorrer todas (ou a maioria) das características das habilidades predominantes de cada estilo (quadro 1), uma vez que se torna possível o aprimoramento do aprendizado, considerando as particularidades predominantes em cada estudante.

\subsection{COMPARAÇÃO DOS ESTILOS DE APRENDIZAGEM DOS DIS- CENTES E DOCENTES}

No gráfico 2, apresentado a seguir, é possível observar a congruência entre os estilos de aprendizagem dos discentes e dos docentes.

Gráfico 2 - Estilos de aprendizagem (Docentes x Discentes)

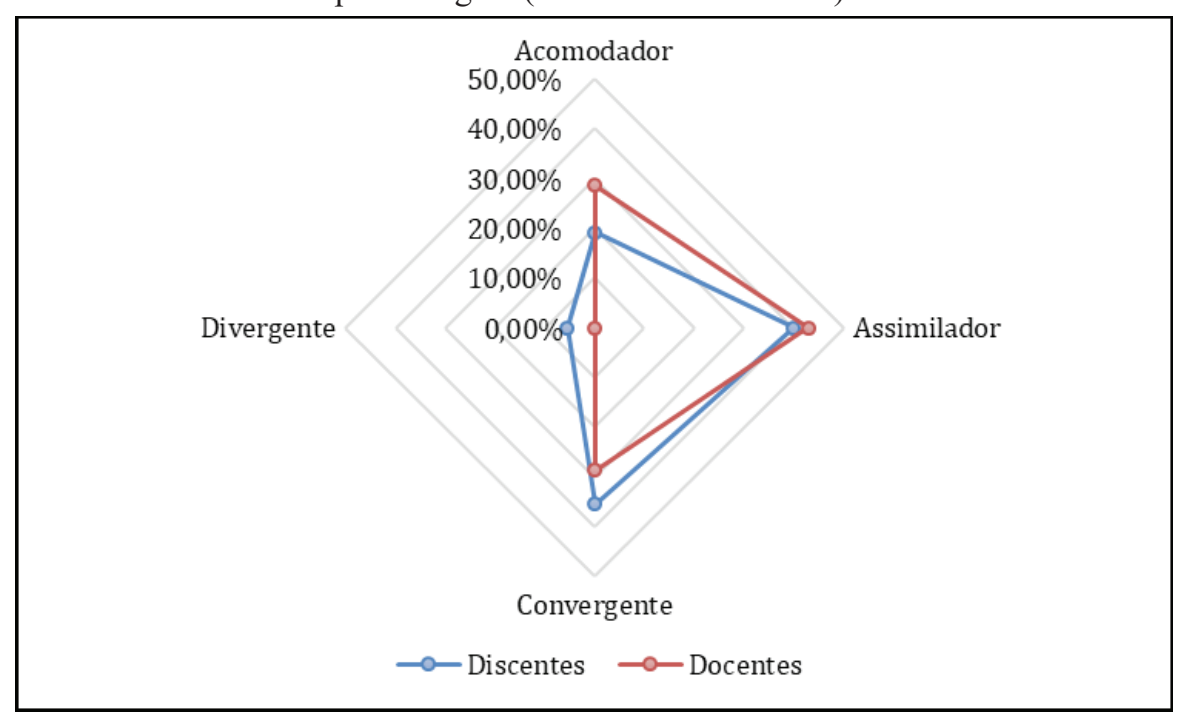

Fonte: elaborado pelos autores (2019).

Percebe-se que há boa relação entre a distribuição dos estilos de aprendizagem quando se analisam os estilos dos docentes e discentes. Ou seja, a forma como se distribui o perfil de aprendizagem de docentes e discentes na instituição é aproximada, sendo predominante em ambos os grupos, o estilo Assimilador. Avalia-se essa aproximação dos estilos como positiva, tendo em vista que proporciona sintonia entre docentes e discentes no processo de ensino e aprendizagem.

Importa destacar que a UPE - Campus Salgueiro é uma universidade pequena, o que possibilita maior fluidez no acompanhamento das mudanças, diferente de grandes estruturas educacionais, em que o fluxo de informações pode ser mais lento. Essa constatação foi fei- ta também por Valente, Abib e Kusnik (2007) ao estudarem a Universidade Estadual de Ponta Grossa (UEPG), que tem o mesmo porte do campus em estudo.

Assim, espera-se que os resultados desta pesquisa possam ser utilizados pela gestão acadêmica, estimulando o corpo docente a conduzir suas aulas na perspectiva de favorecer os estilos de aprendizagem dos discentes. Positivamente, constata-se que os docentes e discentes possuem o mesmo modo de aprender, o que equaliza um modo de ensino e aprendizagem compatível com as necessidades da comunidade acadêmica. No entanto, ampliar para outras estratégias de ensino, considerando os demais estilos de aprendizagem pode favorecer o ensino e a aprendizagem de todos. 


\section{CONSIDERAÇÕES FINAIS}

Estudar os estilos de aprendizagem dos discentes é uma maneira de tentar entender como o professor deve criar sua estratégia de abordagem didática em sala de aula, a fim de aprimorar o processo de ensino e aprendizagem. Assim, esta pesquisa teve como objetivo identificar o estilo de aprendizagem predominante entre os estudantes dos cursos de Bacharelado em Administração e Tecnologia em Logística, ambos da Universidade de Pernambuco - Campus Salgueiro, que é uma unidade ainda de pequeno porte, com cerca de 150 (cento e cinquenta) alunos matriculados.

Constatou-se que o estilo de aprendizagem predominante entre discentes e docentes é o Assimilador. Apesar de o resultado se diferenciar do que aponta a teoria, na medida em que foram encontrados estilos de aprendizagem iguais entre os discentes e os docentes, acredita-se que o alinhamento entre os estilos pode fornecer a equalização do processo de ensino e aprendizagem no Campus. Assim, entendese que tal resultado avança no campo teórico e aponta para uma semelhança do processo de ensino e aprendizagem em uma instituição de pequeno porte.

Contudo, os dados aqui apresentados não podem ser generalizados para outras situações, porém servem como parâmetro para análise em contextos semelhantes. Sugerem-se como estudos futuros: (1) realizar uma pesquisa de corte longitudinal com os discentes de uma turma para observar o processo de amadurecimento e se este influencia no estilo de aprendizagem, questionando-se: acontecem mudanças nos estilos com o decorrer dos anos (maturidade)?; (2) aplicar novo instrumento para saber as preferências dos discentes pela metodologia dos professores, comparando se o estilo de aprendizagem semelhante ao da turma que foi escolhida; (3) realizar uma pesquisa de natureza qualitativa sobre o autoconhecimento de discentes e docentes em relação a seu estilo de aprendizagem, para assim comparar o estilo desejado com o real; (4) em uma ou mais tur- mas, separar alguns grupos de atividades avaliativas com estilos de aprendizagem iguais e outros diferentes, com o intuito de verificar se a combinação ou diferença deles interfere no processo de aprendizagem e trabalho em equipe; e (5) relacionar as notas dos estudantes com seus estilos de aprendizagem predominantes, realizando estudos a partir de técnicas de estatística inferencial a fim de apontar se existe alguma relação entre o estilo de aprendizagem predominante e o desempenho acadêmico e, se sim, qual estilo consegue obter melhores notas.

No contexto prático, propõe-se que a gestão da Universidade possa montar um laboratório de análise do processo de ensino e aprendizagem, e, por meio deste, estudar os elementos apontados aqui como estudos futuros.

Ademais, salienta-se que reconhecer e refletir sobre o estilo de aprendizagem é proveitoso tanto para os estudantes como para os professores. $\mathrm{O}$ discente identifica seu ato de conhecer, tornando-se mais autônomo no processo de aprendizagem e adota estratégias de estudo de acordo com seu estilo de aprendizagem. Da mesma maneira, o docente se beneficia desse reconhecimento porque reflete sobre sua prática e amplia seu olhar para os diferentes estilos de aprendizagem que existem na sala de aula. As ações pedagógicas podem ser reorganizadas visando atender à pluralidade existente no âmbito acadêmico.

Por fim, considerar todas as fases do ciclo de aprendizagem proposto por Kolb possibilita ao docente experimentar novas alternativas de ensino, que não apenas aquelas relacionadas com seu estilo de aprendizagem, mas ampliar e proporcionar um espaço de ensino e aprendizagem capaz de considerar a diversidade e a harmonia necessárias para o ensinar e o aprender.

\section{REFERÊNCIAS}

BRANDÃO, J. M. F. Princípios andragógicos e fatores mediadores da aprendizagem na educação a distância em administração pública. 2014. Dissertação (Mestrado em Administração) - Universidade Federal da Paraíba 
(UFPB), João Pessoa, 2014.

CERQUEIRA, T. C. S. Estilos de aprendizagem em universitários. 2000. Tese (Doutorado em Educação) - Universidade Estadual de Campinas, Campinas, 2000.

CCMD-UFPB - Curso de Comunicação em Mídias Digitais da Universidade Federal da Paraíba. Inventário de Estilo de Aprendizagem de Kolb. Disponível em: http://www.cchla. ufpb.br/ccmd/aprendizagem/. Acesso em: 21 jul. 2019.

CORDEIRO, R. A.; SILVA, A. B. da. Os estilos de aprendizagem influenciam o desempenho acadêmico dos estudantes de finanças? Revista de Administração da Universidade Federal de Santa Maria, v. 5, n. 2, p. 243-261, 2012.

DIAS, G. P. P.; SAUAIA, A. C.; YOSHIZAKI, H. T. Y. Estilos de Aprendizagem Felder Silverman e o Aprendizado com Jogos de Empresa. ERA, São Paulo, v. 53, n. 5, p. 469-484, set./ out. 2013.

DUNN, R.; DUNN, K. Teaching students through their individual learning styles: a practical approach. Reston, VA: Reston Publishing Co., 1978.

FELDER, R. M.; SILVERMAN, L. K. Learning styles and teaching styles in engineering education. International Journal of Engineering Education, Ontario, v. 78, n. 7, p. 674-681, 1988.

FELDER, R. M.; SOLOMAN, B. A. Index of learning styles questionnaire. North Carolina State University, 1991.

FLEMING, N. D.; MILLS, C. Não outro inventário, em vez de um catalizador para a reflexão. [S.l.]: LINCOLN UNIVERSITY, 1992.
KOLB, David A. Experiential learning: experience as the source of learning and development. New Jersey: Prentice-Hall, 1984.

MOREIRA, C. E. R.; MUNCK, L. Estilos de aprendizagem versus treinamento vivencial ao ar livre. Revista de Administração da Universidade Federal de Santa Maria, v. 3, n. 1, p. 9-25, 2010.

OLIVEIRA, P. H. P. de.; BOUZADA, M. A. C. A influência dos estilos de aprendizagem de Kolb sobre a experiência de alunos de graduação em administração no contexto das simulações empresariais. Revista da Universidade Vale do Rio Verde, v. 16, n. 1, 2018.

OLIVEIRA, A. J. et al. Estilos de aprendizagem e estratégias ludopedagógicas: percepções no ensino da contabilidade. Advances in Scientific and Applied Accounting, [S.l.], p. 236262, dez. 2013. ISSN 1983-8611.

PENA, A. F. R.; CAVALCANTE, B.; MIONI, C. C. A Teoria De Kolb: análise dos estilos de aprendizagem no curso de administração da Fecap. Revista Liceu On-Line, v. 5, n. 1, p. 64-84, 2015.

REIS, L. G. D.; PATON, C.; NOGUEIRA, D. R. Estilos de aprendizagem: uma análise dos alunos docurso de ciências contábeis pelo método Kolb. Enfoque Reflexão Contábil, v. 31, n. 1, p. 53-66, 2012.

SILVA, A. B. et al. Dimensões de um sistema de aprendizagem em ação para o ensino de administração. Revista Administração: Ensino e Pesquisa, v. 13, n. 1, p. 9-41, 2012.

SCHMITT, C. S.; DOMINGUES M. J. C. S. Estilos de aprendizagem: um estudo comparativo. Avaliação, Campinas, Sorocaba, SP, v. 21, n. 2, p. 361-385, jul. 2016.

SOUZA, G. H. S. et al. Estilos de aprendizagem dos alunos versus métodos de ensino dos 
professores do curso de administração. In: ENCONTRO DA ANPAD, 37., 2013, Rio de Janeiro. Anais [...]. Rio de Janeiro: ANPAD, 2013.

TREVELIN, A. T. C. Estilos de aprendizagem de Kolb: Estratégias para a melhoria do ensino -aprendizagem. Revista de Estilos de Aprendizaje, v. 4, n. 7, 2011.

VALENTE, N. T. Z.; ABIB, D. B.; KUSNIK, L. F. Análise dos estilos de aprendizagem dos alunos e professores do curso de graduação em ciências contábeis de uma universidade pública do estado do Paraná com a aplicação do inventário de David Kolb. Contabilidade Vista \& Revista, v. 18, n. 1, p. 51-74, 2007. 